\title{
Role of the environmental spectrum in the decoherence and dephasing of multilevel quantum systems
}

\author{
T. Hakioğlu and Kerim Savran \\ Department of Physics, Bilkent University, Bilkent, 06800 Ankara, Turkey \\ (Received 25 October 2004; published 25 March 2005)
}

\begin{abstract}
We examine the effect of multilevels on decoherence and dephasing properties of a quantum system consisting of a nonideal two level subspace, identified as the qubit, and a finite set of higher energy levels above this qubit subspace. The whole system is under interaction with an environmental bath through a CaldeiraLeggett type coupling. The model that we use is an rf-SQUID under macroscopic quantum coherence and coupled inductively to a flux noise characterized by an environmental spectrum. The model interaction can generate dipole couplings which can be appreciable between the qubit and the high levels. The decoherence properties of the qubit subspace is examined numerically using the master equation formalism of the system's reduced density matrix. We calculate the relaxation and dephasing times as the spectral parameters of the environment are varied. We observe that, these calculated time scales receive contribution from all available frequencies in the noise spectrum (even well above the system's resonant frequency scales) stressing the dominant role played by the nonresonant transitions. The relaxation and dephasing and the leakage times thus calculated, strongly depend on the appreciably interacting levels determined by the strength of the dipole coupling. Under the influence of these nonresonant and multilevel effects, the validity of the two level approximation is dictated not by the low temperature as conveniently believed, but by these multilevel dipole couplings as well as the availability of the environmental modes.
\end{abstract}

DOI: 10.1103/PhysRevB.71.115115

\section{INTRODUCTION}

Currently a large number of model approaches are present for formulating the decoherence phenomena in the literature. The original Caldeira-Leggett model ${ }^{1}$ is based on a quantum system under the influence of a double well tunneling potential with a linear coupling to an infinite bath of harmonic oscillators. If the potential is sufficiently smooth and the separation between the qubit and the high energy levels is well above the environmental temperature, this original model is normally represented as a two level system ${ }^{2}$ (2LS) interacting with the bosonic environment (spin-boson model). An incomplete list of this wide literature is provided in Refs. 3-5. Another popular model of decoherence is the central spin system in which central 2LS couples to a large number of environmental two level systems. The pros and cons of these two rival models have been extensively studied. ${ }^{5}$

Realistically, and aside from the genuine 2LS, a large majority of physical systems suggested as qubit is far from being ideal and include some number of higher energy levels. Higher levels in a multileveled system (MLS) can be effective in the dynamics in two ways. The first is the leakage of the information from qubit subspace to higher levels which can happen in the presence of some uncontrollable coupling to a noise field with a sufficiently wide spectrum. Populating higher levels is basically thought to be a manifestation of resonant transitions at long times. On the other hand, for an arbitrary and wide noise spectrum, the same type of systemnoise coupling can also induce nonresonant transitions which affect the short time dynamics of the interaction and contribute to the decoherence times. In the coupling of a multileveled system to a wide environmental spectrum both effects should therefore be expected. As a consequence of
PACS number(s): 72.90.+y, 03.65.Yz, 03.67.Lx, 85.25.Dq

these physical effects, and including the leakage factor, the validity of the analytic techniques devised to approximate these MLS in terms of simpler two-level model may become critically questionable.

In this work, we look for the answers of the following basic questions: (a) Can one understand the effect of the higher levels on decoherence in a MLS under interaction with an environment? and (b) what is the role played by the environmental spectrum in the two-leveledness of a MLS?

The MLS can itself be manifestly $N$-leveled or a truncated approximation of a larger system with much higher number of levels. Examples of both cases have been well known. For the former, organic molecules with certain discrete rotational symmetries and low energy configurations of single polymerized chains are good examples. The vibrational energy spectra of atoms and molecules are good examples for the latter. We remark however, that a concise treatment of the decoherence effects based on such MLS has not been developed yet. Realistic MLS can be found for instance in superconducting systems in the macroscopic quantum coherence regimes. In this work, we use an rf-SQUID in the flux regime to generate our model system Hamiltonian for a multileveled quantum system. In the interaction with an environmental noise we use two scales which parametrize the low and high frequency sectors of the noise spectrum.

In Sec. II we give an introduction of the model MLS. There we concentrate on the properties of the environmentally induced dipole matrix elements between the levels. Section III recalls the reduced density matrix (RDM) master equation formalism and adopts it for the coupling of the MLS to the environment. The noise correlator and the system-noise kernel, are defined in Sec. III. The results are presented together with the calculations for pure 2LS (in Sec. III A) to allow a comparison with the earlier work. Most original results of the paper are included in Sec. III. The 
MLS with three or higher levels are examined in Sec. III B separately for the cases $N=3, N=4$, and $4<N$. There, we confirm the leakage effects and observe that the short time nonresonant transitions dominate the decoherence times. As a consequence of these short time effects, we demonstrate in Sec. III C that, nonresonant transitions introduce severe restrictions in the validity of the two level approximation.

\section{THE MODEL MULTILEVEL SYSTEM}

The majority of the accepted methods (particularly the influence functional) widely used in the literature is applicable to the two-leveled dynamics. The results are generally believed to be true for 2LS and at temperatures well below the energy separation of the qubit and the high levels. ${ }^{1,2}$ Such two level approximations are tempting since they allow explicit analytic expressions for the decoherence times as functions of the system's parameters. Exact methods are also available on pure $2 \mathrm{LS}{ }^{4}$

If one does not take for granted the validity of such approximations, a direct investigation of the MLS-environment interaction must be made. In a MLS a need for explicit dependence on number of levels and their mutual couplings arises. To keep these dependencies, we represent here the MLS in its eigenenergy basis.

Our MLS is an rf-SQUID operating under macroscopic quantum coherence conditions given by the dimensionless Hamiltonian

$$
\mathcal{H}_{s} /\left(\hbar \Omega_{0}\right)=\frac{1}{2}\left[-\partial_{\varphi}^{2}+\left(\varphi-\varphi_{\text {bias }}\right)^{2}\right]+\beta \cos (\gamma \varphi),
$$

where $\Omega_{0}=2 \pi / \sqrt{L C}$ is the harmonic frequency with $L$ being the inductance of the SQUID loop and $C$ is the effective capacitance of the Josephson junction, $\beta=E_{J} / \hbar \Omega_{0}$ is the dimensionless ratio of the Josephson energy $E_{J}$ to the harmonic energy, $\gamma=\hbar \sqrt{L / C}\left(2 \pi / \Phi_{0}\right)^{2}$ is a dimensionless scale parameter, $\varphi_{\text {bias }}=2 \pi \Phi_{\text {bias }} / \Phi_{0}$ is the effective bias in the flux (applicable in a current biased junction), and $\varphi=2 \pi \Phi /\left(\gamma \Phi_{0}\right)$ is the flux $(\Phi)$ dependent dimensionless phase (here $\Phi_{0}$ $=h c / 2 e$ is the superconducting flux quantum). The Hamiltonian (1) is clearly an infinite level system. Truncating the eigenspace at $N$ levels, it becomes, in the energy basis

$$
\mathcal{H}_{s}=\sum_{n=0}^{N-1} E_{n}(\zeta)|\zeta, n\rangle\langle\zeta, n|,
$$

where $\zeta$ describes the set of system parameters $\Omega_{0}, \beta, \gamma, \varphi_{\text {bias }}$ where $E_{n}(\zeta)$, and $|\zeta, n\rangle$ are, respectively, the parameter dependent eigenenergies and eigenvectors of the MLS. This set of parameters is sufficiently general to accommodate a variety of possible effects including the degeneracy of the lowest two levels (the qubit subspace), the symmetry of the wave functions, etc. [we define the degeneracy factor by $\eta=\left(E_{2}\right.$ $\left.-E_{1}\right) /\left(E_{1}-E_{0}\right)$ for MLS and $\eta_{2}=\left(E_{1}+E_{0}\right) /\left(E_{1}-E_{0}\right)$ for 2LS]. These parameters $\Omega_{0}, \beta, \varphi_{\text {bias }}$ control, respectively, the high energy harmonic, the low energy anharmonic spectra and the reflection symmetry of the rf-SQUID potential, respectively. At low energies, a simple numerical diagonalization of (1) reveals that there are low lying eigenenergy con-

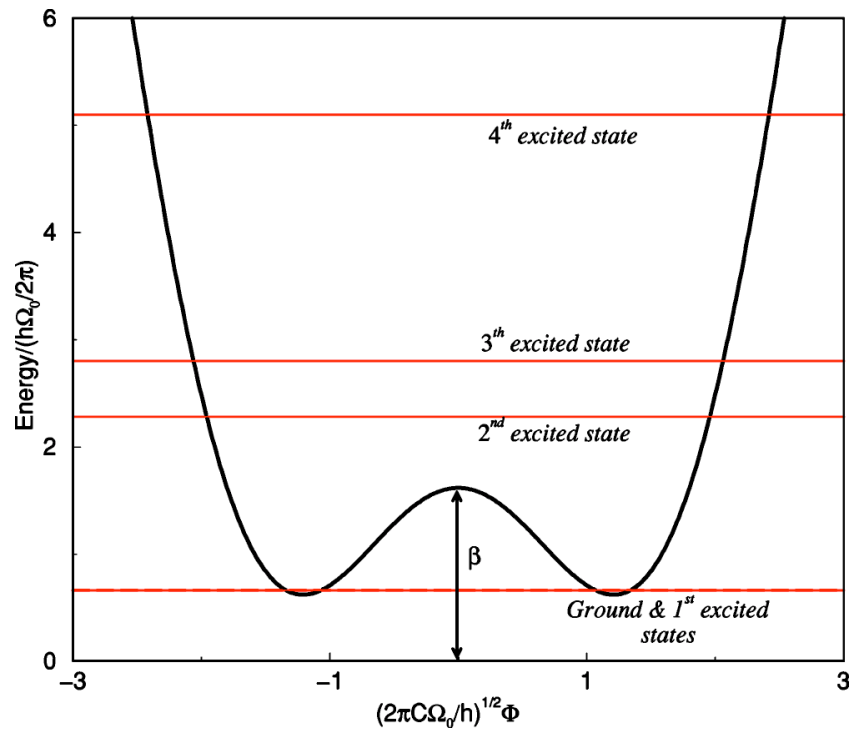

FIG. 1. The double-well potential and the eigenenergy configurations corresponding to the singly degenerate (SD) case. Here the numerical values of the dimensionless parameters for this SD configuration are $\beta \simeq 1.616$ and $\gamma \simeq 1.753$. The harmonic energy scale and the degeneracy factors are, respectively, $\hbar \Omega_{0}=10^{-3} \mathrm{eV}$ and $\eta_{2} \simeq 10^{7}$.

figurations within the double well regime in which the SQUID potential is strongly anharmonic. An interesting case here is to find highly degenerate ${ }^{6}$ levels corresponding to the first two eigenstates for the symmetric double-well potential (i.e., $\varphi_{\text {bias }}=0$ ). This particular case has been extensively examined previously for $2 \mathrm{LS}$ using semiclassical methods with an arbitrarily weak tunneling between the wells., ${ }^{2,3}$ Another configuration that turns out to be important in our calculations is the doubly degenerate (DD) configuration for systems with $4 \leqslant N$ in which the first four levels are pairwise degenerate with large degeneracy factors. The double-well potential and energies corresponding to both $\mathrm{SD}$ and $\mathrm{DD}$ configurations which are often used in the paper, are shown in Figs. 1 and 2, respectively.

The rf-SQUID is shown to be a convenient (and nonunique) model for studying multilevel effects due to the fact that the transitional dipole couplings between the qubit subspace and the higher levels are non-negligible as shown in Fig. 3. Any other physical Hamiltonian with similar features would qualify for a model MLS.

In the rest of the paper the harmonic frequency $\Omega_{0}$ $=2 \pi / \sqrt{L C}$ is a free parameter that we use for scaling energy and time. ${ }^{6}$

Coupling to noise: The system-noise interaction is considered to be of Caldeira-Leggett type linear coupling between the SQUID's macroscopic flux $\hat{\varphi}$ and the environmental flux $\hat{\varphi}_{e}$ arising from the finite inductance of the SQUID loop. This coupling can be expanded in terms of the environmental modes as $\hat{\varphi}_{e}=\Sigma_{k} \eta_{k}\left(\hat{b}_{-k}^{\dagger}+\hat{b}_{k}\right)$ where $k$ is the mode index. The system noise interaction is simply $\mathcal{H}_{\text {int }}=(\alpha / 2) \hat{\varphi} \hat{\varphi}_{e}$ where $\alpha$ represents the strength of the inductive coupling ( $\alpha$ is to be normalized by $\hbar \Omega_{0}$ ). The interaction Hamiltonian is given by 


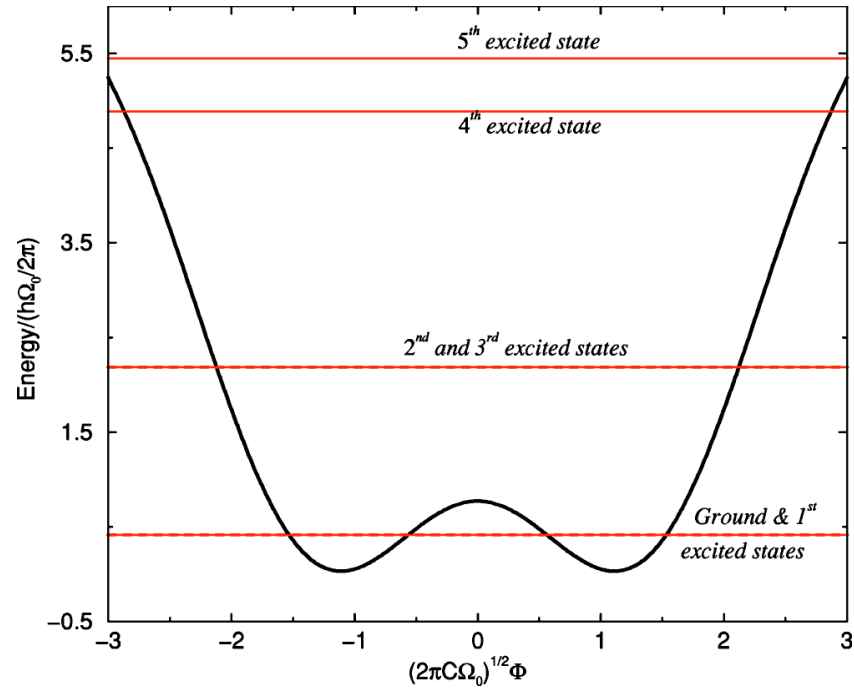

FIG. 2. The double-well potential and the eigenenergy configurations corresponding to the doubly degenerate (DD) case. The numerical values of the dimensionless parameters corresponding to this DD configuration are $\beta \simeq 0.772$ and $\gamma \simeq 2.187$. The harmonic energy scale and the degeneracy factors are $\hbar \Omega_{0}=10^{-3} \mathrm{eV}, \eta_{2}, \eta$ $\simeq 10^{6}$.

$$
\mathcal{H}_{\text {int }}=\frac{\alpha}{2} \sum_{r, s=0}^{N-1}(\varphi)_{r s}|\zeta, s\rangle\langle\zeta, r| \hat{\varphi}_{e}
$$

Here $(\varphi)_{r s}=\langle\zeta, r|\hat{\varphi}| \zeta, s\rangle$ are the noise induced dipole matrix elements. For the model MLS described by (1), the dipole matrix is real and symmetric.

The rf-SQUID provides a sufficiently general example of a multileveled system by the finite dipole transition matrix elements for both the symmetric (i.e., $\varphi_{\text {bias }}=0$ ) and asymmetric (i.e., $\varphi_{\text {bias }} \neq 0$ ) potential configurations. In the symmetric case even parity transitions vanish which results in manifestly off-diagonal system-noise coupling. Physically, this is

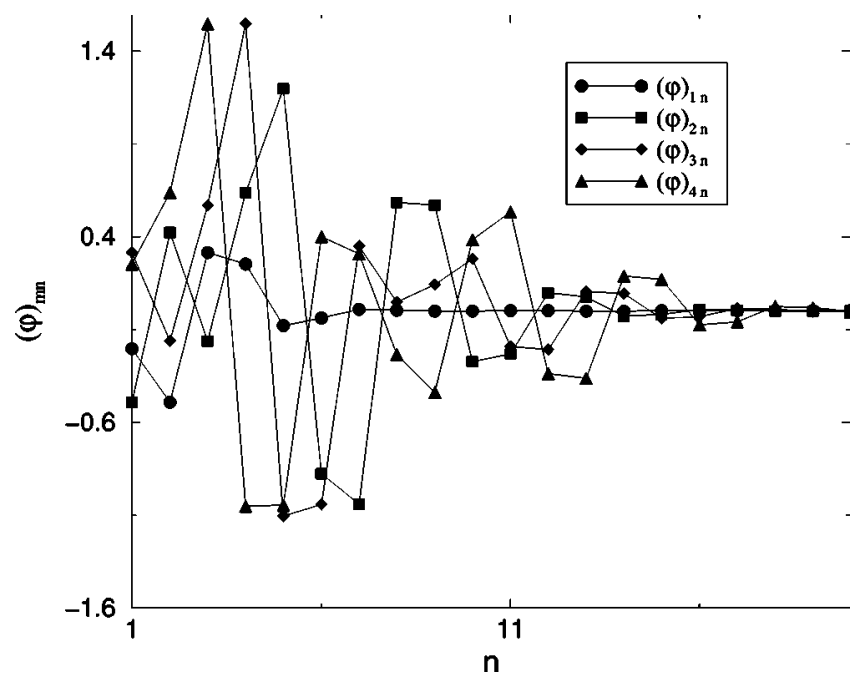

FIG. 3. A few nonzero dipole matrix elements $(\varphi)_{n m}$ of the coupling of the rf-SQUID to a flux noise versus the level index [calculated in the eigenenergy basis of (1) for an asymmetric potential].

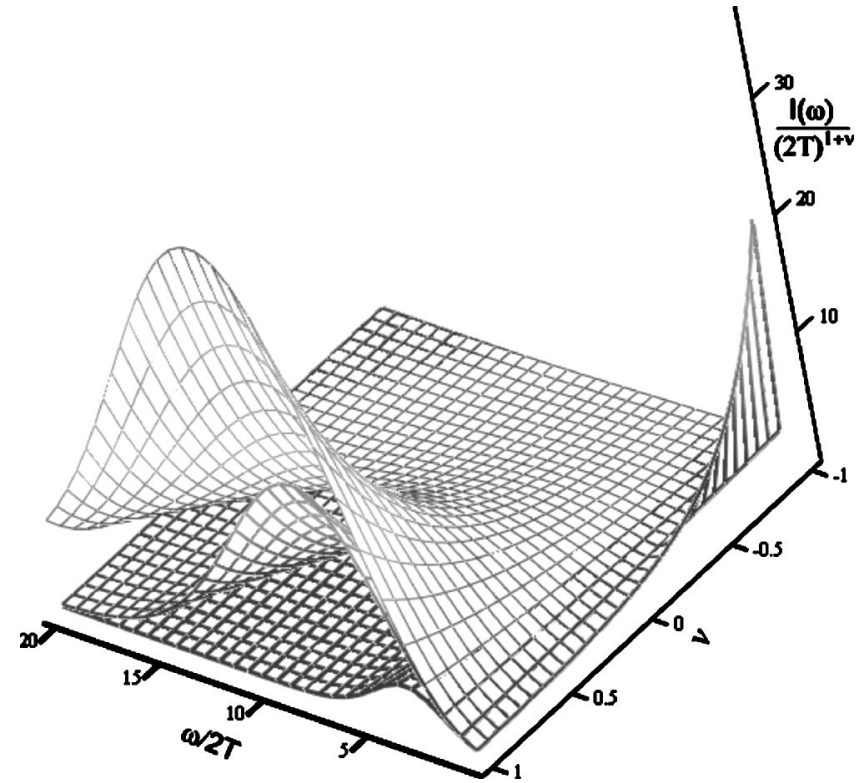

FIG. 4. The variation of $I(\omega)$ in (4) versus $\omega$ and $\nu$ for $-1 \leqslant \nu$ $\leqslant 1$ parametrized by $\Lambda / T=10,50,50,100$ (from the innermost to the outermost surfaces, respectively).

in contrast to the most popular models used in the literature. On the other hand, when the potential is tilted, the parity restriction is lifted by which diagonal system-noise couplings are also generated.

In Fig. 3 the noise induced couplings for an asymmetric potential corresponding to $\varphi_{\text {bias }}=0.01$ is plotted as a function of the relevant level indices interacting with the dipole coupling. Data indicate that the induced dipole strengths between the qubit and the higher energy states are comparable to those among the qubit states. Therefore, the high energy transitions cannot be trivially ignored. The high energy transitions normally appear as a result of the resonant interactions with the high energy sector of the noise spectrum under long interaction times. However, in the reduced system these transitions appear in the short time dynamics as well, and the short time dynamics is dominated by the nonresonant processes. Since decoherence is dominantly affected by the short time behavior, the nonresonant processes should have observable effects on decoherence. Indeed we observe these effects in the solution of the master equation (Secs. III A-III C).

We next consider the environmental spectrum. Regarding this, we take a thermal Gaussian with a low frequency tail obeying a power law as

$$
I(\omega)=\omega^{1+\nu} e^{-\omega^{2} / 4 \Lambda^{2}} \operatorname{coth}(\omega / 2 T),
$$

where $\Lambda$ is the effective noise cutoff frequency and $\nu$ describes the subohmic (i.e., $\nu<0$ ), superohmic (i.e., $\nu>0$ ), and ohmic (i.e., $\nu=0$ ) character of the spectrum. These two parameters define the high and low frequency character of the noise field as mentioned in the Introduction. (See. Fig. 4.)

If the resonant processes were dominant in the noiseinduced transitions, one would then only concentrate on that part of the spectrum where $\omega$ would be close to a relevant 
transition energy $\Delta E$. For $-1 \lesssim \nu$ (subohmic), the following two regions are of particular importance: (a) at sufficiently low temperatures and high cutoff corresponding to $T \ll \Lambda$, the dominant mechanism of relaxation is through spontaneous de-excitations, ${ }^{7}$ we call this region region-I; (b) at high temperatures and high cutoff the region $\omega \ll \min (\Lambda, T)$ provides a wider range of strong environmental couplings which we call region II. If the character of the spectrum is more like ohmic or superohmic, i.e., $\nu \simeq 0$ or $\nu \simeq 1$, respectively, the availability of the low frequency modes is not so high. Therefore, in the ohmic and superohmic regimes, region II would dominate the relaxation/dephasing (RD) phenomena. Hence it is concluded that, if the resonant processes were dominant, one would maintain a sufficiently low environmental temperature to largely eliminate the decoherence effects for ohmic and superohmic cases.

Our calculations in this work however demonstrate that decoherence cannot be avoided at zero temperature and the decoherence times are influenced not by the strength of the spectral coupling at the resonant transitions but by the whole spectral range.

\section{MASTER EQUATION AND THE REDUCED DENSITY MATRIX FOR THE MLS}

In the study of decoherence due to the weak environmental influence, one conventional way is to calculate the time dependent RDM by solving the master equation. This formalism has been known since the works of Bloch, Redfield, and Fano $(\mathrm{BRF})^{8}$ and widely applied to the current decoherence problems for which many standard references exist. ${ }^{9}$ The standard BRF formalism assumes fully Markovian conditions for the solution of the master equation, which leads to analytically solvable results for $2 \mathrm{LS} .^{10}$ However, this assumption is not free of drawbacks which was explored originally in Ref. 11 and lately in Ref. 12 as well as in Ref. 13 in the context of spin magnetic resonance and relaxation.

The time evolution of the RDM is obtained by

$$
-i \hbar \frac{d}{d t} \hat{\tilde{\rho}}(t)=\left[\hat{\widetilde{\rho}}(t), \tilde{\mathcal{H}}_{\mathrm{int}}(t)\right],
$$

where the tilde denotes the interaction picture. In the context of decoherence, we will give more emphasis on the exponential time scales in the solution of (5). A convenient way to proceed is then to apply the Born-Oppenheimer approximation in which the full density matrix is initially a product of the system and environmental ones [i.e., $\quad \hat{\tilde{\rho}}(0)=\hat{\tilde{\rho}}^{(S)}(0)$ $\left.\otimes \hat{\tilde{\rho}}_{e}(0)\right]$ and at any later and sufficiently short time approximately separates as $\hat{\tilde{\rho}}(t)=\hat{\widetilde{\rho}}^{(S)}(t) \otimes \hat{\tilde{\rho}}_{e}(0)$.

The iterative solution of (5) including the second order in the interaction with the partial trace performed over the environmental degrees of freedom yields the master equation for the RDM,

$$
\frac{d}{d t} \widetilde{\rho}_{n m}^{(S)}(t)=-\int_{0}^{t} d t^{\prime} \sum_{r, s} K_{r s}^{n m}\left(t, t^{\prime}\right) \widetilde{\rho}_{r s}^{(S)}\left(t^{\prime}\right),
$$

in which we adopt the model interaction Hamiltonian (3) for the system-noise kernel. This kernel is found to be

$$
\begin{aligned}
K_{r s}^{n m}\left(t, t^{\prime}\right)= & \frac{\alpha^{2}}{4}\left\{\mathcal{F}\left(t-t^{\prime}\right)\left[\left(\hat{\widetilde{\varphi}}_{t} \hat{\tilde{\varphi}}_{t^{\prime}}\right)_{n r} \delta_{s, m}-\left(\hat{\widetilde{\varphi}}_{t^{\prime}}\right)_{n r}\left(\hat{\tilde{\varphi}}_{t}\right)_{s m}\right]\right. \\
& \left.+\mathcal{F}^{*}\left(t-t^{\prime}\right)\left[\left(\hat{\tilde{\varphi}}_{t^{\prime}} \hat{\tilde{\varphi}}_{t}\right)_{m s} \delta_{r, n}-\left(\hat{\tilde{\varphi}}_{t}\right)_{n r}\left(\hat{\tilde{\varphi}}_{t^{\prime}}\right)_{s m}\right]\right\} .
\end{aligned}
$$

Here $\mathcal{F}\left(t-t^{\prime}\right)=\mathcal{F}^{*}\left(t^{\prime}-t\right)$ is the complex noise correlation function,

$$
\mathcal{F}\left(t-t^{\prime}\right)=\mathcal{T} r_{e}\left[\hat{\tilde{\varphi}}_{e}(t) \hat{\tilde{\varphi}}_{e}\left(t^{\prime}\right) \rho_{e}(0)\right]=\left\langle\hat{\widetilde{\varphi}}_{e}(t) \hat{\tilde{\varphi}}_{e}\left(t^{\prime}\right)\right\rangle
$$

and

$$
\hat{\tilde{\varphi}}_{t}=\sum_{k, \ell=0}^{N-1}(\hat{\varphi})_{k \ell} e^{-i\left(E_{k}-E_{\ell}\right) t}|\zeta, k\rangle\langle\zeta, \ell|
$$

is the dipole operator in the interaction picture. Expanding the noise field $\hat{\varphi}_{e}$ in the independent harmonic modes and calculating (8) in thermal equilibrium one obtains the standard thermal noise correlator,

$$
\begin{aligned}
\mathcal{F}\left(t-t^{\prime}\right)= & 2 \sum_{k} \eta_{k}^{2}\left[\operatorname{coth}\left(\omega_{k} / 2 T\right) \cos \omega_{k}\left(t-t^{\prime}\right)\right. \\
& \left.-i \sin \omega_{k}\left(t-t^{\prime}\right)\right] .
\end{aligned}
$$

The noise spectrum is assumed to be continuous of which the real part is responsible for RD effects and is given by the spectral density in (4). In the numerical calculations we include the noise correlation function as ${ }^{15}$

$$
\begin{aligned}
\mathcal{F}\left(t-t^{\prime}\right)= & 2 \int_{0}^{\infty} d \omega \omega^{1+v} e^{-\omega^{2} / 4 \Lambda^{2}}\left[\operatorname{coth}(\omega / 2 T) \cos \omega\left(t-t^{\prime}\right)\right. \\
& \left.-i \sin \omega\left(t-t^{\prime}\right)\right] .
\end{aligned}
$$

Inserting (11) in (7) we obtain the system-noise kernel for our model. A numerical upper frequency cutoff of $\omega_{\max }$ $=5 \Lambda$ is used in the numerical integral in (11).

The solution of (6) is determined in the weak systemnoise interaction limit by the competition of three time scales: $\tau_{B}$, noise correlation time scale, $\tau_{R}$ and $\tau_{D}$, the relaxation and dephasing time scales ${ }^{14}$ of the reduced system, respectively. The noise correlation time scale is found roughly from the thermal Gaussian bath spectral width as $\tau_{B} \simeq 1 / \Lambda$. The RD time scales are found by fitting the envelope in the solution of (6) to the decaying exponential ${ }^{3}$

$$
\left|\rho_{i j}(t)\right| \simeq\left|\rho_{i j}(\infty)\right|+\left[\left|\rho_{i j}(0)\right|-\left|\rho_{i j}(\infty)\right|\right] \exp \left(-t / \tau_{i j}\right),
$$

by the formula

$$
\tau_{i j}^{-1} \simeq-\left.\frac{1}{1-\left|\rho_{i j}(\infty) / \rho_{i j}(0)\right|} \frac{d \ln \left|\rho_{i j}\right|}{d t}\right|_{t=0},
$$

where in (12) and (13), $i=j=1$ is used in the calculation of the relaxation rate $\left(\tau_{R}^{-1}\right)$ and $i=0, j=1$ is used for the qubit dephasing rate $\left(\tau_{D}^{-1}\right)$. For the RDM at asymptotic times we have $\rho_{11}(\infty)=1 / N$ and $\left|\rho_{10}(\infty)\right|=0$. The equation (13) breaks down when $\left|\rho_{i j}(0)\right|=\left|\rho_{i j}(\infty)\right|$ which we stay away from by appropriately choosing $\rho_{i j}(0)$.

In this work, the numerical solution of (6) is performed by discretizing time in steps of $\Delta t=10^{-2} \Omega_{0}^{-1}$ (i.e., $t=n \Delta t, t^{\prime}$ 


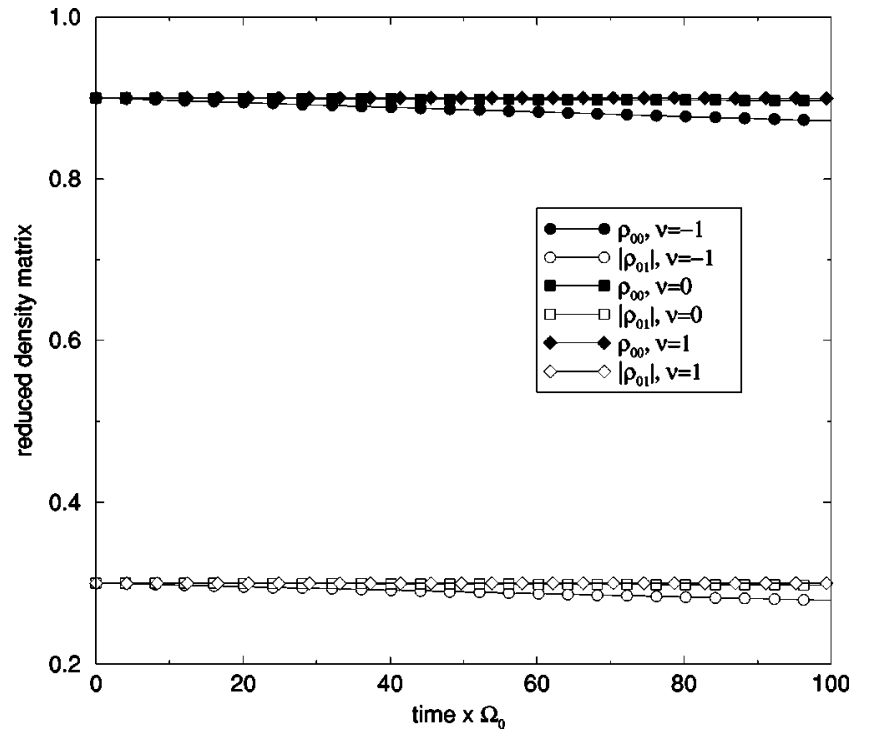

FIG. 5. Time dependence of the RDM in units of $\Omega_{0}^{-1}$ for various representative $\nu$ parameter sets at $\Lambda=0.1$. For the model system the potential is symmetric and the bare $2 \mathrm{LS}$ is in SD configuration.

$=m \Delta t$ ). The time sum is then performed at each external step given by a fixed $n$ in the discrete triangular region $m \leqslant n$ using the standard nonadaptive Euler algorithm. The Hermiticity and the normalization of the RDM at each time step is controlled within a relative accuracy of $10^{-25}$. All calculated energies as well as time scales (particularly the RD times) are also given in units of $\hbar \Omega_{0}$ and $\Omega_{0}^{-1}$, respectively. Moreover, we consider zero temperature $(T=0)$ results except one specific case in Sec. III C and $\alpha=0.01$ throughout the paper.

\section{A. RDM solutions for the $2 \mathrm{LS}$}

The solution of the 2LS is shown in Fig. 5 for a few representative environmental parameters and for the SD configuration. The degeneracy parameter is $\eta_{2} \sim 10^{6}$.

The first observation is that, the results indicate that the relaxation time scale $\tau_{R}$ (read from the filled symbols) and the dephasing time scale $\tau_{\text {dep }}$ (read from the hollow symbols) are compatible. This result is in agreement particularly with the recent exact $2 \mathrm{LS}$ calculations using the path integral influence functional formalism. ${ }^{4}$ As the asymptotic time behavior is concerned, for symmetric configurations (pure $\sigma_{x}$ coupling), the density matrix converges to the maximum entropy (informationless) limit $\hat{I} / 2$, where $\hat{I}$ is the unit matrix, irrespective of the spectral properties of the noise or the systemnoise coupling.

We observe that all regions in the noise spectrum have strong influence on RD phenomenon. For this observation, one must compare Figs. 5-7 corresponding to different spectral widths. For instance, as shown in Fig. 5, for $\Lambda=0.1$, we recover the under damped and weak dephasing limit of Ref. 3 for all $\nu$.

A curious observation in Figs. 5-7 is the strong dependence of RD time scales on the spectral width $\Lambda$. If the resonant processes were dominant, one would naively expect that at very small temperatures, the resonant excitations will

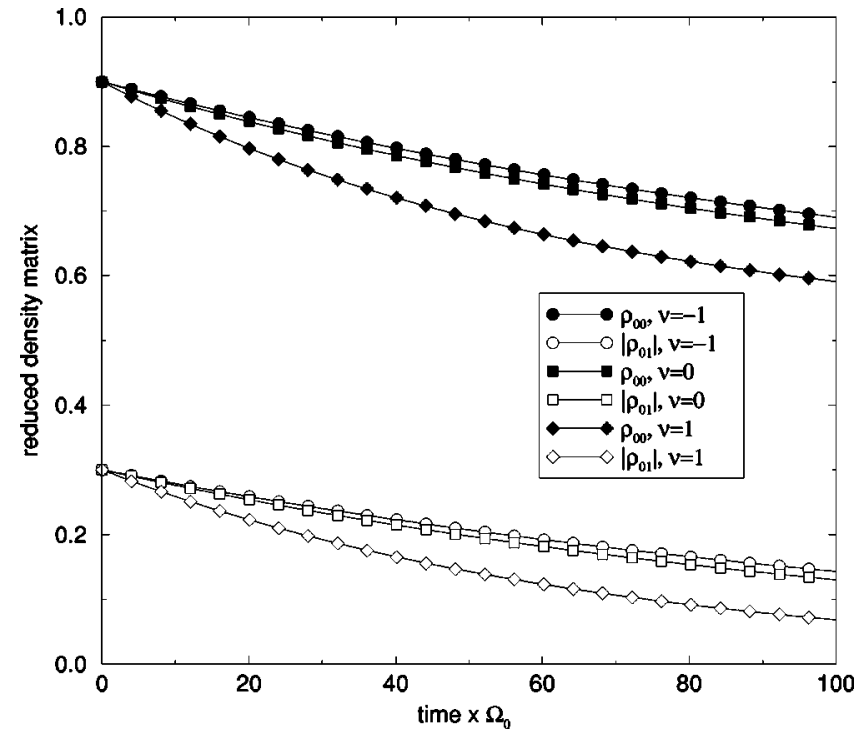

FIG. 6. Time dependence of the RDM in the SD configuration with respect to $\nu$ parameter. The width is taken as $\Lambda=1$.

be unfavored and the decoherence would be suppressed. The point that is often neglected in this popular argument is the contribution of the short time nonresonant transitions covered by the whole spectrum. The resonant transitions are favored when the system interacts with the environment at sufficiently large times. The system however relaxes differently at short times by preferring to stay off-resonant in its interaction with the noise field thereby sampling all regions of the noise spectrum. This causes the strong dependence on $\Lambda$ we observe at short times.

The major difference of the model interaction Hamiltonian in (3) from the standard ( $\sigma_{z}$-type) spin-boson model is in the manipulation of the potential. In contrast to the standard spin-boson model, in our case only nondiagonal, $\sigma_{x}$, type coupling is present under the symmetric potential (see

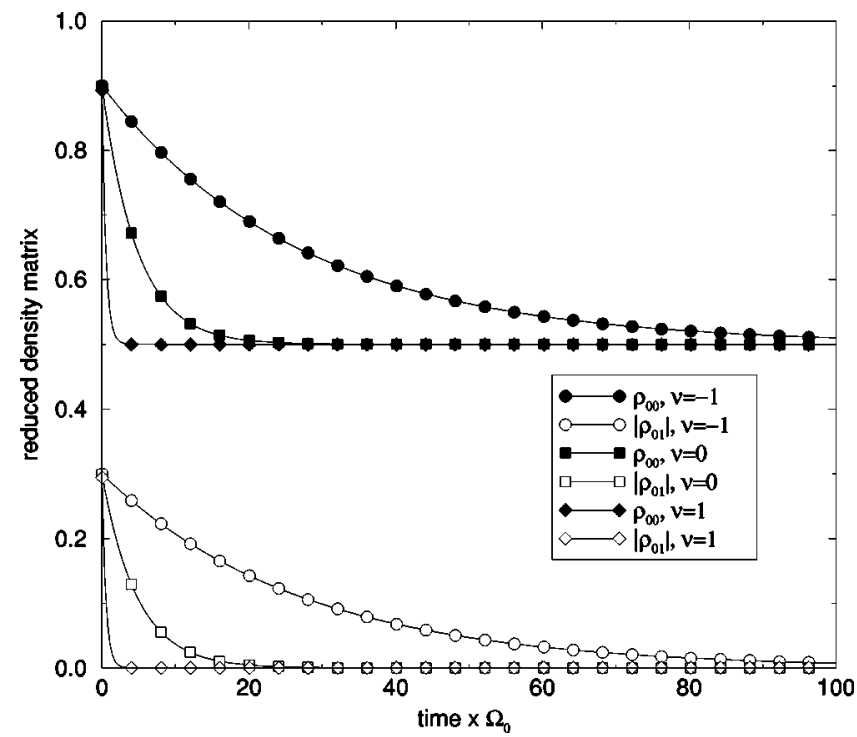

FIG. 7. The $\nu$ behavior of the RDM is shown for a symmetric potential. The noise parameters are $\Lambda=5$ and $\nu=-1,0,1$. 


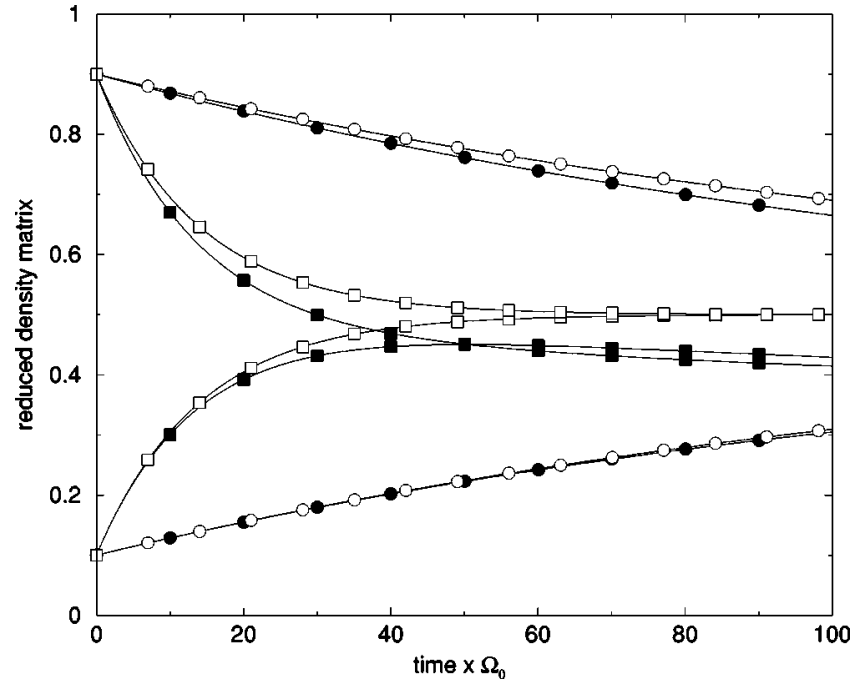

FIG. 8. Comparison of the effect of the spectral width $\Lambda$ on the time dependence of the elements $\rho_{00}$ and $\rho_{11}$ between the $2 \mathrm{LS}$ and 3LS. We use $\nu=-1$. The open symbols refer to the case $N=2$ and the solid symbols refer to $N=3$. More specifically, circle is $\Lambda=1$, square is $\Lambda=10$.

Fig. 3). As a result, dramatic differences in the time dependence of the reduced system are observed between the two models. For instance, the diagonal coupling is standardly considered for the study of pure dephasing. In this type of coupling the relaxation is manifestly forbidden and the initial states do not change their populations. The diagonal coupling also yields strongly temperature dependent dephasing rates with the rates vanishing at $T=0$. On the other hand, when the system-noise coupling is not diagonal, the induced transitions between the system states can probe the entire noise spectrum creating decoherence even at zero temperature. These induced transitions are nonresonant and they should have observable effects on decoherence. ${ }^{16}$ The RD times observed as the result of such system-noise coupling are expected to be nonzero even at zero temperature. This characteristic behavior of the nondiagonal coupling is confirmed in our calculations both for the 2LS in Figs. 5-7 and for the MLS in the following sections. Recently, there are other claims using realistic models on decoherence effects in mesoscopic systems ${ }^{17}$ as well as a few experimental confirmations on the saturation of the RD rates at low temperatures. ${ }^{18}$

\section{B. MLS with $3 \leqslant N$}

As $N$ increases the complexity of the formal methods devised for $2 \mathrm{LS}$, such as the noninteracting blip approximation, ${ }^{3}$ increases at each time step as $N^{2}$ rendering the analytic sum over all virtual configurations in the path integral approach intractable. We have already observed that there are major pitfalls in the two-level approximation. For instance, the dependence of the decoherence rates for a $2 \mathrm{LS}$, as shown in Figs. 5-7, on the off-resonant energy scales such as $\Lambda$ has been overseen in this approximation. Therefore the short time behavior of a decohering system-which is the most prominent regime in the quantum computational

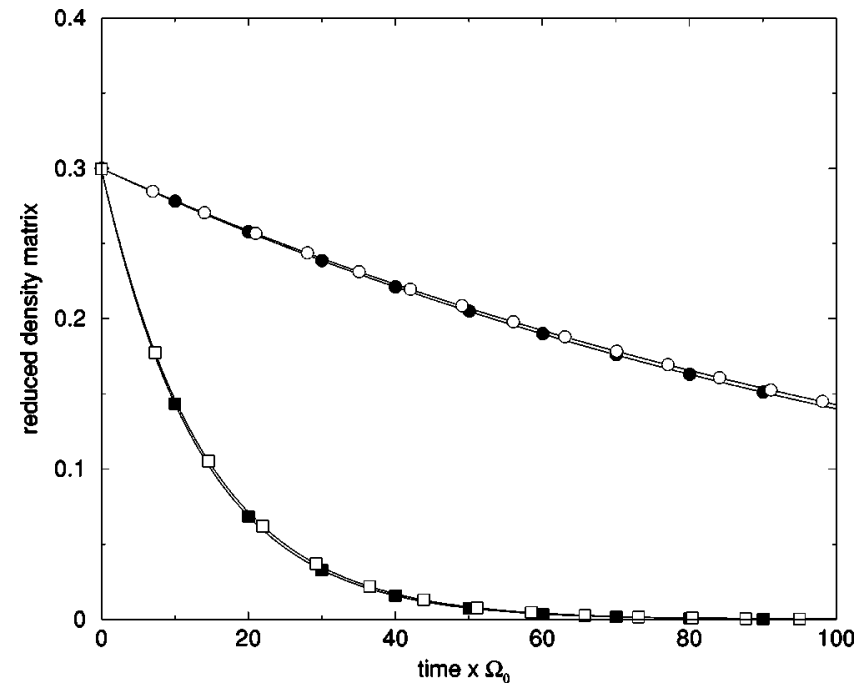

FIG. 9. Comparison of the effect of the spectral width $\Lambda$ on the time dependence of $\left|\rho_{01}\right|$ between the 2LS and 3LS. The parameters are the same as in Fig. 8.

perspective-is affected by a large frequency region in the noise spectrum. Hence the high excitation levels of the quantum system are vulnerable to leakage and dephasing effects induced by nonresonant short time (energy nonconserving) processes. We now study the decoherence of the simplest multilevel extension of a $2 \mathrm{LS}$.

\section{1. $N=3$ case}

From the quantum computational point of view, the three level systems can be as important as the two level ones. Recently, we proposed a method to perform the $q$-gate operations by radiation-free couplings within a three level system. $^{19}$

The solution of (6) for $N=3$ are shown in Figs. 8 and 9, compared to the $N=2$ case at $\Lambda=1,10$. We look at the symmetric potential in the SD configuration. For 3LS, the observed energies form a $\Lambda$-shaped configuration and, in units of $\Omega_{0}$, are roughly $E_{0} \simeq E_{1} \approx 0.1$ and $E_{2} \approx 2.3$. The degeneracy parameter $\eta=\left(E_{2}-E_{1}\right) /\left(E_{1}-E_{0}\right) \simeq 10^{6}$ and the third level $E_{2}$ is above the double well barrier as shown in Fig. 1.

When $\Lambda<E_{2}-E_{1}$ the resonant coupling of the first two levels to the third level is very weak. At short observational times, the significant contribution should therefore come from the nonresonant excitations. As $\Lambda<E_{2}-E_{1}$ however, these nonresonant transitions are also suppressed by the Gaussian cutoff. As a result, the 3LS is basically confined to its highly degenerate qubit subspace. For $E_{2}-E_{1}<\Lambda$ the third level is allowed to participate in the transitions. A significant leakage should therefore be found in the $N=3$ case in comparison with the $N=2$ (shown in Fig. 8 for $\Lambda=1,10$ at $\nu=-1$ ). However, the dephasing rates are unaffected (shown in Fig. 9).

\section{2. $N=4$ case}

We compare the 4LS with a 2LS in Figs. 10 and 11 for $\nu=-1$. In Fig. 10 the $4 \mathrm{LS}$ is compared to $2 \mathrm{LS}$ when both systems are in SD configuration. 


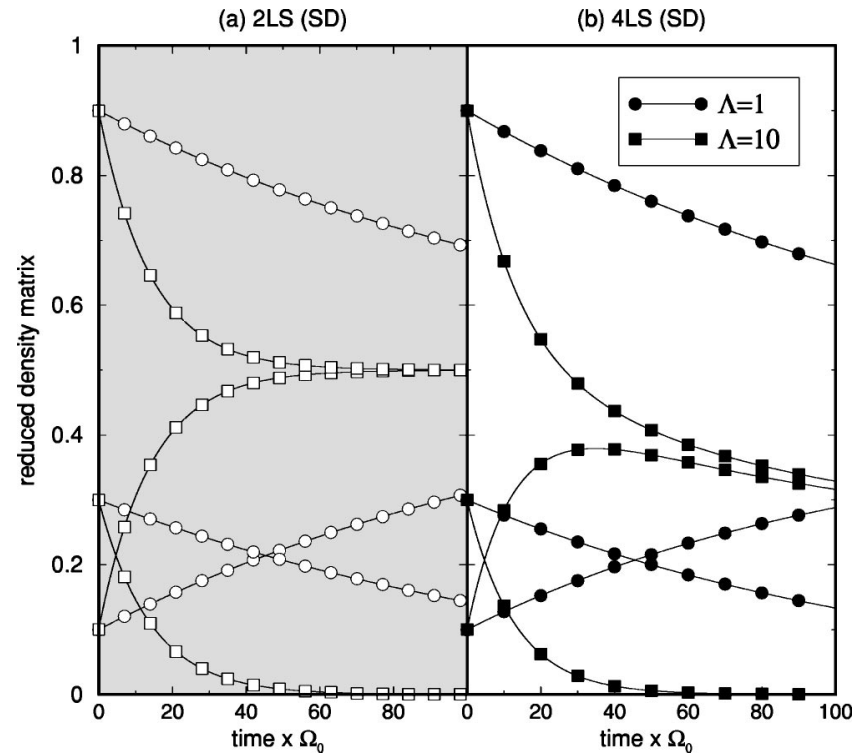

FIG. 10. Comparison, at $\nu=-1$, of the effect of the spectral width $\Lambda$ on the time dependence of the elements $\rho_{00}, \rho_{11}$, and $\left|\rho_{01}\right|$ between the $2 \mathrm{LS}$ and $4 \mathrm{LS}$. The symbols are the same as in Figs. 8 and 9 . The figure describes the singly degenerate case $\eta \simeq 10^{6}$.

For the singly degenerate (SD) case, the qualitative features between the 2LS and the 4LS are similar to the previously discussed case between 2LS and the 3LS. Here, we observe for the diagonal elements, a much higher relaxation rate (as well as leakage) out of the qubit subspace during the observed time for the 4LS although a much less significant change is observed in the dephasing rates. For higher spectral widths such as $\Lambda=30$ (not included in the figures) dephasing rates are also significantly different.

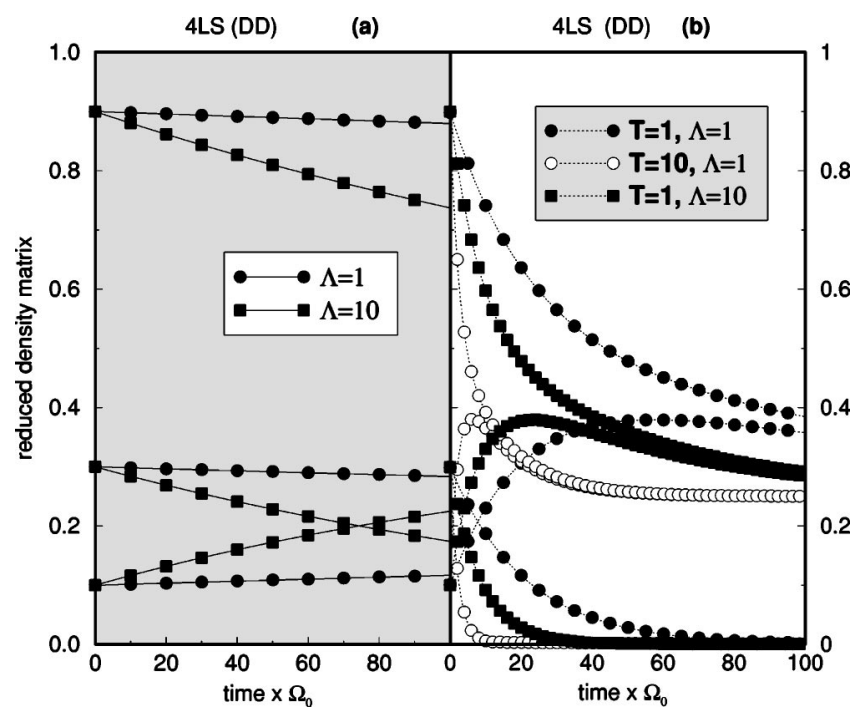

FIG. 11. (a) The rates at zero temperature and $\nu=-1$ for the doubly degenerate configuration $\eta=\left(E_{2}-E_{1}\right) /\left(E_{1}-E_{0}\right) \simeq 3 \times 10^{6}$ and $\eta_{\mathrm{DD}}=\left(E_{3}-E_{2}\right) /\left(E_{2}-E_{1}\right) \simeq 10^{6}$. The curves indicate that relaxation, dephasing, and leakage are greatly suppressed. (b) The thermal case at the indicated $\Lambda, T$ values at $\nu=-1$.

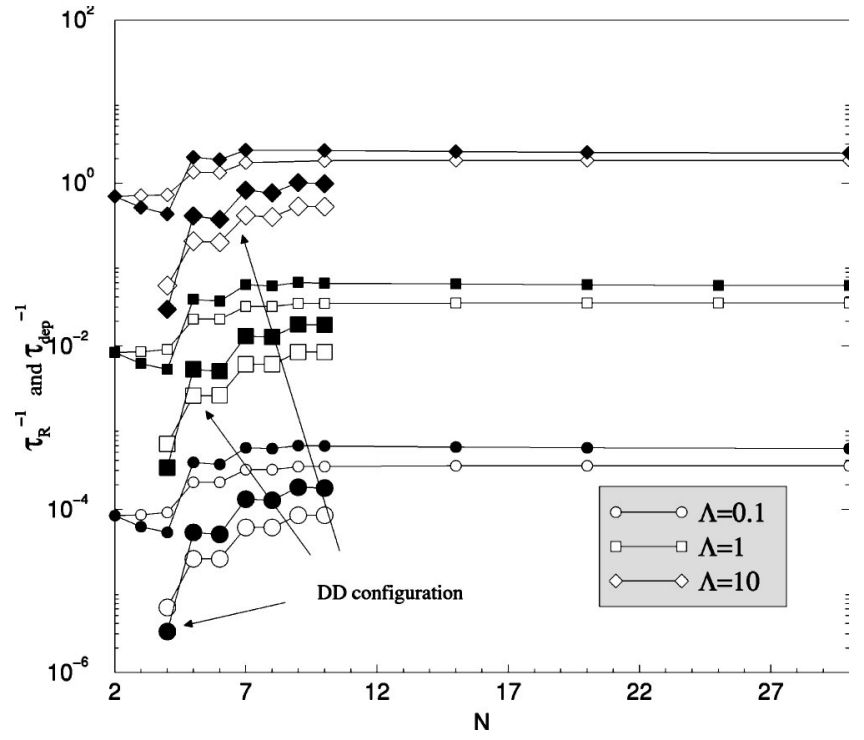

FIG. 12. Relaxation and dephasing rates against the number of levels for different spectral widths at $T=0$ and $\nu=0$. Note the logarithmic vertical axis. Small symbols refer to the singly degenerate MLS and the larger symbols refer to the doubly degenerate one. The open and solid symbols refer to dephasing and relaxation times, respectively.

In Fig. 11(a) the four level system is in DD (see Fig. 2 configuration). The second set of curves is plotted in Fig. 11(b) corresponding to the DD in Fig. 11(a) but with environmental modes in equilibrium at finite temperatures. In DD configuration we define two degeneracy factors as $\eta=\left(E_{2}\right.$ $\left.-E_{1}\right) /\left(E_{1}-E_{0}\right)$ and $\eta_{\mathrm{DD}}=\left(E_{3}-E_{2}\right) /\left(E_{2}-E_{1}\right)$ where $\eta \simeq 3$ $\times 10^{6}$ and $\eta_{\mathrm{DD}} \simeq 10^{6}$. A strong suppression is observable (by almost two orders of magnitude) in the RD rates at zero temperature as shown in Fig. 11(a). However the rates strongly depend on the temperature.

\section{Arbitrary $N$ case}

In Fig. 12 the data are represented at zero temperature and $\nu=0$. Three different curves stand for (bottom to top) $\Lambda$ $=0.1,1,10$ with the open symbols corresponding to dephasing and the solid ones to the relaxation rates. Each set of data is shown for $\mathrm{SD}$ as well as DD configurations separately. Also note that the vertical axis is logarithmic.

Let us concentrate first on the SD configurations in Fig. 12. In a large $\Lambda$ range $N=4$ and $N \sim 10$ appear to be two crucial points. For $4<N$ relaxation is approximately twice faster than dephasing and both rates rapidly saturate near $N$ $\simeq 10$ and they are independent of $N$ for $10<N$. The onset of saturation is naturally model dependent. In our case this onset coincides with the range of strong dipole transition matrix elements of the model in (1) (see Fig. 3). Turning to the DD configurations, we observe that for the same environmental parameters and for all $N$, decoherence rates for the DD case are strongly suppressed by nearly two orders of magnitude as compared to the SD configuration. Currently we do not know the reason behind this strong suppression of decoherence rates in doubly degenerate systems. 

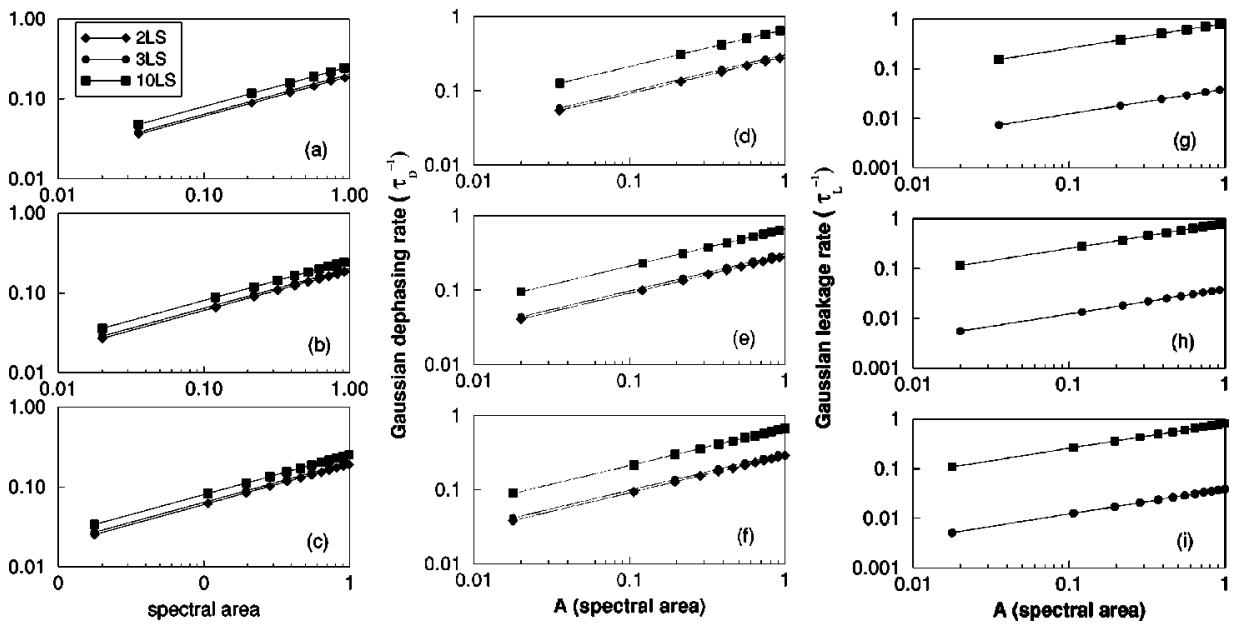

\section{Nonresonant short-time effects in the Gaussian regime}

An important conclusion that we inferred during the preceding sections is the role of nonresonant transitions on decoherence at short time scales. The nonresonant effects are already visible in the exponentially time dependent region as shown there. Nevertheless, to predict the influence of the nonresonant processes more precisely, one must go to shorter time scales than the exponential region where the time behavior is Gaussian. The solution of (5) yields Gaussian envelope at sufficiently short times as $\hat{\tilde{\rho}}(t) \sim \exp \left(-t^{2} / \tau_{G}^{2}\right) \hat{\tilde{\rho}}(0)$ where $\tau_{G}$ describes either the relaxation type or dephasing type Gaussian short time scale. We show in Figs. 13(a)-13(f) these Gaussian rates $\left(\tau_{G}^{-1}\right)$ versus the spectral area for various $\nu$ values. Curves corresponding to different $\nu$ coincide completely, demonstrating that it is the combined areal dependence that matters. Moreover, the log-log plots indicate the power law $\tau_{G} \sim \sqrt{A}$ where $A$ is the area under the spectral function $I(\omega)$ as given by $A=\int_{0}^{\infty} d \omega I(\omega)$. This scaling indicates the strong and unbiased influence of all frequencies in the noise spectrum and is an evidence of the existing nonresonant frequencies in the transitions between the multilevels.

In Figs. 13(g)-13(i) we depict the Gaussian leakage rates versus spectral area for $3 \leqslant N$. The Gaussian leakage rates measure how rapidly the quantum information escapes into the higher levels demonstrating that the effect of the nonresonant transitions is not a mere renormalization ${ }^{3}$ of the two level subsystem but a genuine multilevel effect.

\section{CONCLUSIONS}

In this work, we examined the decoherence properties of a general multilevel system interacting with an environmental noise spectrum using non-Markovian master equation formalism.

With regard to the two basic questions in the Introduction which motivated this work, we have that (a) it is mainly the nonresonant transitions which affect the short time dynamics of a quantum system interacting with an environment. At short observation times, the multileveledness is unavoidable if there is finite coupling between the levels; and, if the noise interacting with the system is sufficiently wide to allow for nonresonant transitions. (b) The nonresonant transitions increase the decoherence rates practically without saturation. For generic MLS, the decoherence rates increase with increasing nonresonant effects. The leakage effect is more pronounced for a quantum system with higher number of levels and interacting with a wide spectral noise.

Here another general observation is that, decoherence in MLS is more pronounced than that in the pure 2LS. A distinct counterexample to this is presented in the doubly degenerate MLS with $4 \leqslant N$. The RD rates are found to be suppressed by two orders of magnitude in comparison with the singly degenerate or nondegenerate systems for the same parameter set. As a side remark, we believe that understanding the true nature of this strong suppression in DD systems may be a crucial development in building low-decoherence qubits.

The decoherence is caused by interaction driven fluctuations where the number of excitations in the environment fluctuates. Similar zero temperature decoherence mechanisms have been recently verified for dephasing in the mesoscopic persistent current rings experimentally ${ }^{18}$ and studied theoretically. ${ }^{20-22}$ In particular, the saturation observed in the dephasing time has been argued in favor of the zero temperature intrinsic quantum fluctuations.

Finally we conclude that, the two-level approximation is not well-satisfied by conditions solely determined by the temperature. Our study here suggests that the validity of the two-level approximation should be bestowed upon the environmental spectrum but not on the sufficiently low temperatures. It is also possible to examine these effects by using much simpler models with different environmental spectra. Our current effort is devoted to an elaborate criticism of two leveledness using such models. ${ }^{23}$

\section{ACKNOWLEDGMENTS}

This research is supported by the Scientific and Technical Research Council of Turkey (TÜBİTAK) Grant No. TBAG2111 (101T136). The authors thank I. O. Kulik and E. Mese for critical comments. 
${ }^{1}$ A. O. Caldeira and A. J. Leggett, Phys. Rev. Lett. 46, 211 (1981); Ann. Phys. (N.Y.) 149, 374 (1983).

${ }^{2}$ A. J. Leggett and Anupam Garg, Phys. Rev. Lett. 54, 857 (1985).

${ }^{3}$ A. J. Leggett, S. Chakravarty, A. T. Dorsey, Matthew P. A. Fisher, Anupam Garg, and W. Zwerger, Rev. Mod. Phys. 59, 1 (1987); Daniel Loss and David P. DiVincenzo, Int. J. Mod. Phys. B 17, 5489 (2003); Till Vorrath, Tobias Brandes, and Bernhard Kramer, cond-mat/304118 (unpublished).

${ }^{4}$ Charis Anastopoulos and B. L. Hu, Phys. Rev. A 62, 033821 (2000).

${ }^{5}$ M. Dube and P. C. E. Stamp, Quantum Physics of Open Systems [Chem. Phys., Special issue 268, 257 (2001)]; cond-mat/ 0102156; P. C. E. Stamp and I. S. Tupitsyn, cond-mat/0308139; Leonid Fedichkin, Akrady Fedorov, and Vladimir Privman, Proc. SPIE 5105, 243 (2003).

${ }^{6}$ In a finite double well potential there is no manifest degeneracy. However highly degenerate configurations can be obtained in the vicinity of level crossings. It can be numerically shown [see for instance T. Hakioglu, J. Anderson, and F. Wellstood, Phys. Rev. B 66, 115324 (2002)] that at the level crossings the symmetric and antisymmetric configurations are highly confined either within the left or the right wells. The tunneling matrix element is proportional to the overlap of these configurations which is minimized at the level crossings.

${ }^{7} \mathrm{We}$ assume that the system is initially prepared at a given superposition state $\psi(0)=a|0\rangle+b|1\rangle$ where $|0\rangle$ and $|1\rangle$ are, respectively, the ground and the first excited states in the qubit subspace.

${ }^{8}$ F. Bloch, Phys. Rev. 102, 104 (1956); A. G. Redfield, IBM J. Res. Dev. 1, 19 (1957); U. Fano, Phys. Rev. 96, 869 (1954).

${ }^{9}$ Michele Governale, Milena Grifoni, and Gerd Shön, Chem. Phys. 208, 273 (2001); Guido Burkard, Roger H. Koch, and David P. DiVincenzo, Phys. Rev. B 69, 064503 (2004); Yuriy Makhlin, Gerd Schön, and Alexander Shnirman, New Dimensions in Mesoscopic Physics, edited by R. Fazio, V. F. Gantmakher, and Y.
Imry (Kluwer, Dordrecht, 2003), pp. 197-236

${ }^{10}$ Anatoly Yu. Smirnov, Phys. Rev. B 67, 155104 (2003), and the third reference in Ref. 9.

${ }^{11}$ P. N. Argyres and P. L. Kelley, Phys. Rev. 134, 98 (1964).

${ }^{12}$ A. Suarez, R. Silbey, and I. Oppenheim, J. Chem. Phys. 97, 5101 (1992).

${ }^{13}$ M. Grifoni, E. Paladino, and U. Weiss, Eur. Phys. J. B 10, 719 (1999).

${ }^{14}$ H. P. Breuer and F. Petruccione, The Theory of Open Quantum Systems (Oxford University Press, Oxford, 2002).

${ }^{15}$ B. L. Hu, Juan Pablo Paz, and Yuhong Zhang, Phys. Rev. D 45, 2843 (1992).

${ }^{16}$ Florian Marquardt and C. Bruder, Phys. Rev. B 65, 125315 (2002).

${ }^{17}$ D. S. Golubev and A. D. Zaikin, Phys. Rev. Lett. 81, 1074 (1998); Phys. Rev. B 62, 14061 (2000).

${ }^{18}$ P. Mohanty, E. M. Q. Jariwala, and R. A. Webb, Phys. Rev. Lett. 78, 3366 (1997).

${ }^{19}$ I. O. Kulik and T. Hakioğlu, Eur. Phys. J. B 30, 219 (2002); also recently, a Markovian Linblad approach was used for the RDM of a multilevel system in connection with the quantum Zeno effect [see for instance, D. Bruno, P. Facchi, S. Longo, P. Minelli, S. Pascazio, and A. Scardicchio, quant-ph/0206143 (unpublished)].

${ }^{20}$ D. Natelson, R. L. Willett, K. W. West, and L. N. Pfeiffer, Phys. Rev. Lett. 86, 1821 (2001).

${ }^{21}$ Pascal Cedraschi, Vadim V. Ponomarenko, and Markus Büttiker, Phys. Rev. Lett. 84, 346 (2000); M. Büttiker, Decoherence from Vacuum Fluctuations, Proc. Electronic Correlations: From Meso- to Nano-Physics, edited by G. Montambaux and T. Martin (EDP Sciences, Les Ulis, 2001), pp. 231-236; see also condmat/0105519 (unpublished).

${ }^{22}$ J. J. Lin and N. Giordano, Phys. Rev. B 35, 1071 (1987); D. M. Pookr et al., J. Phys.: Condens. Matter 1, 3289 (1989).

${ }^{23}$ K. Savran, T. Hakioğlu, and E. Mese, J. Phys. B (to be published). 